\title{
Euler Type Integrals and Integrals in Terms of Extended Beta Function
}

\author{
Subuhi Khan ${ }^{1}$ and Mustafa Walid Al-Saad ${ }^{2}$ \\ ${ }^{1}$ Department of Mathematics, Aligarh Muslim University, Aligarh 202002, India \\ ${ }^{2}$ General Requirements Unit, Fatima College of Health Sciences, Al Ain, UAE \\ Correspondence should be addressed to Subuhi Khan; subuhi2006@gmail.com
}

Received 15 February 2014; Accepted 12 May 2014; Published 12 June 2014

Academic Editor: Alfredo Peris

Copyright ( 2014 S. Khan and M. W. Al-Saad. This is an open access article distributed under the Creative Commons Attribution License, which permits unrestricted use, distribution, and reproduction in any medium, provided the original work is properly cited.

We derive the evaluations of certain integrals of Euler type involving generalized hypergeometric series. Further, we establish a theorem on extended beta function, which provides evaluation of certain integrals in terms of extended beta function and certain special polynomials. The possibility of extending some of the derived results to multivariable case is also investigated.

\section{Introduction}

Euler generalized the factorial function from the domain of natural numbers to the gamma function

$$
\Gamma(\alpha)=\int_{0}^{\infty} t^{\alpha-1} e^{-t} d t \quad(\operatorname{Re}(\alpha)>0)
$$

defined over the right half of the complex plane. This led Legendre (in 1811) to decompose the gamma function into the incomplete gamma functions, $\gamma(\alpha, x)$ and $\Gamma(\alpha, x)[1]$,

$$
\begin{gathered}
\gamma(\alpha, x)=\int_{0}^{x} t^{\alpha-1} e^{-t} d t \quad(\operatorname{Re}(\alpha)>0), \\
\Gamma(\alpha, x)=\int_{x}^{\infty} t^{\alpha-1} e^{-t} d t
\end{gathered}
$$

which are obtained from (1) by replacing the upper and lower limits by $x$, respectively. The closed-form solutions to a considerable number of problems in applied mathematics, astrophysics, nuclear physics, statistics, and engineering can be expressed in terms of incomplete gamma functions. These functions develop singularities at the negative integers. Chaudhry and Zubair [2] extended the domain of these functions to the entire complex plane by inserting a regularization factor $\exp (-b / t)$ in the integrand of $(1)$. For $\operatorname{Re}(b)>0$, this factor clearly removes the singularity coming from the limit $t=0$. For $b=0$, this factor becomes unity, and thus we get the original gamma function. We note the following relation [3, page $20(1.2)]$ :

$$
\begin{aligned}
\Gamma_{b}(\alpha) & =\int_{0}^{\infty} t^{\alpha-1} \exp \left(-t-\frac{b}{t}\right) d t \\
& =2(b)^{\alpha / 2} K_{\alpha}(2 \sqrt{b}) \quad(\operatorname{Re}(\alpha)>0),
\end{aligned}
$$

where $K_{n}(x)$ is the modified Bessel function of the second kind of order $n$ (or Macdonald's function) [1]. The relationships between the generalized gamma and Macdonald functions could not have been apparent in the original gamma function. These generalized gamma functions proved very useful in diverse engineering and physical problems; see, for example, $[2,3]$ and references therein.

We note that Riemann's zeta function $\zeta(x)$ defined by the series [1, page $85(2.98)]$

$$
\zeta(x)=\sum_{n=1}^{\infty} \frac{1}{n^{x}} \quad(x>1)
$$

is useful in proving convergence or divergence of other series by means of comparison test. Zeta function is closely related to the logarithm of the gamma function and to the polygamma functions. The regularizer $\exp (-b / t)$ also proved very useful in extending the domain of Riemann's zeta function, thereby providing relationships that could not have been obtained with the original zeta function. 
In view of the fact that the regularization factor is useful in extending the domain of the gamma and zeta functions. The domain of other special functions could be usefully extended in a similar manner.

In particular, Euler's beta function $B(\alpha, \beta)$ has a close relationship to gamma function, that is,

$$
B(\alpha, \beta)=B(\beta, \alpha)=\frac{\Gamma(\alpha) \Gamma(\beta)}{\Gamma(\alpha+\beta)},
$$

and can be usefully extendable. We first recall the basic Euler integral which defines the beta function as follows:

$$
B(\alpha, \beta)=\int_{0}^{1} u^{\alpha-1}(1-u)^{\beta-1} d u \quad(\operatorname{Re}(\alpha), \operatorname{Re}(\beta)>0)
$$

Keeping in view the fact that for the $\alpha-\beta$ symmetry to be preserved there must be symmetry of the integrand in $u$ and $1-u$, Chaudhry et al. [3] introduced an extension of Euler's beta function $B(\alpha, \beta)$ in the following form:

$$
\begin{array}{r}
B(\alpha, \beta ; b)=\int_{0}^{1} t^{\alpha-1}(1-t)^{\beta-1} \exp \left(-\frac{b}{t(1-t)}\right) d t \\
(\operatorname{Re}(b)>0),
\end{array}
$$

which for $b=0$ reduces to the original beta function.

The transformations $t=(u+1) / 2$ and $t=(u-a) /$ $(c-a)$ in $(7)$ yield the following integral representations for the extended beta function (EBF) $B(\alpha, \beta ; b)[3$, page $22(2.9)$, (2.11)]:

$$
\begin{aligned}
& B(\alpha, \beta ; b) \\
& =2^{1-\alpha-\beta} \\
& \quad \times \int_{-1}^{1}(1+u)^{\alpha-1}(1-u)^{\beta-1} \exp \left(-\frac{4 b}{\left(1-u^{2}\right)}\right) d u \\
& \quad(\operatorname{Re}(b)>0),
\end{aligned}
$$

$$
\begin{aligned}
& B(\alpha, \beta ; b) \\
& =(c-a)^{1-\alpha-\beta} \\
& \quad \times \int_{a}^{c}(u-a)^{\alpha-1}(c-u)^{\beta-1} \exp \left(-\frac{b(c-a)^{2}}{(u-a)(c-u)}\right) d u \\
& (\operatorname{Re}(b)>0),
\end{aligned}
$$

respectively.

The EBF $B(\alpha, \beta ; b)$ is extremely useful in the sense that most of the properties of the beta function carry over naturally and simply for it. This extension is also important due to the fact that this function is related to other special functions for particular values of the variables.
We note the following connections [3]:

$$
\begin{aligned}
& B(\alpha,-\alpha ; b)=2 e^{-2 b} K_{\alpha}(2 b) \quad(\operatorname{Re}(b)>0), \\
B(\alpha, \alpha ; b) & \sqrt{\pi} 2^{-\alpha} b^{(\alpha-1) / 2} e^{-2 b} W_{-\alpha / 2, \alpha / 2}(4 b) \\
= & \sqrt{\pi} 2^{1-2 \alpha} e^{-4 b} \Psi\left(\frac{1}{2}, 1-\alpha ; 4 b\right) \quad(\operatorname{Re}(b)>0),
\end{aligned}
$$

where $W_{m, n}(z)$ denotes the Whittaker function [1] and $\Psi(a, c ; z)$ denotes the second form of solutions of Kummer's equation [4].

Also, we note that

$$
\begin{gathered}
B(0,0 ; b)=2 e^{-2 b} K_{0}(2 b) \quad(\operatorname{Re}(b)>0), \\
B\left(\frac{1}{2},-\frac{1}{2} ; b\right)=\sqrt{\frac{\pi}{b}} e^{-4 b} \quad(\operatorname{Re}(b)>0), \\
B\left(\frac{1}{2}, \frac{1}{2} ; b\right)=\pi \operatorname{Erfc}(2 \sqrt{b}) \quad(\operatorname{Re}(b)>0),
\end{gathered}
$$

where $\operatorname{Erfc}(z)=(\sqrt{\pi} / 2) \operatorname{erfc}(z)$ denotes the complementary error function [4].

Explicit evaluations of some integrals of Euler type

$$
\int_{0}^{1} u^{\alpha-1}(1-u)^{\beta-1} f(u) d u
$$

for some particular functions $f$, specially in the symmetric case $\alpha=\beta$, are derived in [5]. These evaluations are related to various reduction formulae for hypergeometric functions represented by such integrals. These formulae generalize the evaluations of some symmetric Euler integrals implied by the following result due to Pitman [6].

If a standard Brownian bridge is sampled at time 0, time 1 and at $n$ independent random times with uniform distribution on $[0,1]$, then the broken line approximation to the bridge obtained from these $n+2$ values has a total variation whose mean square is $n(n+1) /(2 n+1)$.

Motivated and inspired by the work of Ismail and Pitman [5] and Chaudhry et al. [3], in this paper, we derive the evaluations of certain Euler type integrals and some integrals in terms of $\operatorname{EBF} B(\alpha, \beta ; b)$. In Section 2 , we obtain the evaluations of certain integrals of the following type:

$$
\begin{aligned}
& I_{d, \alpha, \beta, \gamma, a, e}[\phi(t), \psi(t)] \\
& =\frac{1}{B(\alpha, \beta)} \int_{a}^{e}(t-a)^{\alpha-1}(e-t)^{\beta-1}(\phi(t))^{\gamma} \\
& \left.\quad \times{ }_{r} F_{t}\left[\begin{array}{l}
\left(f_{r}\right) ; \\
\left(g_{t}\right) ;
\end{array}\right] d \psi(t)\right] d t,
\end{aligned}
$$

where ${ }_{r} F_{t}$ denotes the generalized hypergeometric series [4], for particular functions $\phi(t)$ and $\psi(t)$. In Section 3, we establish a theorem on $\operatorname{EBF} B(\alpha, \beta ; b)$ and apply it to obtain evaluations of certain integrals in terms of $\operatorname{EBF} B(\alpha, \beta ; b)$. Finally, we give some concluding remarks in Section 4. 


\section{Euler Type Integrals}

We derive the evaluations of certain integrals of the following type:

$$
\begin{aligned}
I_{d, \alpha, \beta, \gamma, a, e} & {[\phi(t), \psi(t)] } \\
= & \frac{1}{B(\alpha, \beta)} \int_{a}^{e}(t-a)^{\alpha-1}(e-t)^{\beta-1}(\phi(t))^{\gamma} \\
& \left.\times{ }_{r} F_{t}\left[\begin{array}{l}
\left(f_{r}\right) ; \\
\left(g_{t}\right) ;
\end{array}\right] d \psi(t)\right] d t .
\end{aligned}
$$

We evaluate the integrals of type (15) for particular functions $\phi(t)$ and $\psi(t)$ by considering the following cases.
Case 1. Taking $\phi(t)=\left(1-x_{1} t\right)^{-\alpha_{1}}\left(1-x_{2} t\right)^{-\alpha_{2}}, \psi(t)=1 / t(1-t)$, $a=0$, and $e=\gamma=1$ in (15) and using [5, page 962 (7)]

$$
\begin{array}{r}
\int_{0}^{1} t^{\alpha-1}(1-t)^{\beta-1}\left(1-x_{1} t\right)^{-\alpha_{1}}\left(1-x_{2} t\right)^{-\alpha_{2}} d t \\
=B(\alpha, \beta) F_{1}\left[\alpha, \alpha_{1}, \alpha_{2} ; \alpha+\beta ; x_{1}, x_{2}\right] \\
\left(\operatorname{Re}(\alpha), \operatorname{Re}(\beta)>0 ; \max \left\{\left|x_{1}\right|,\left|x_{2}\right|\right\}<1\right),
\end{array}
$$

where $F_{1}$ denotes the Appell function [4], in the r.h.s. after expanding ${ }_{r} F_{t}\left[\begin{array}{l}{ }_{\left(f_{r}\right) ;} \\ \left(g_{t}\right) ;\end{array} d \psi(t)\right]$ and simplifying, we find the following integral:

$$
\begin{gathered}
I_{d, \alpha, \beta, 1,0,1}\left[\left(1-x_{1} t\right)^{-\alpha_{1}}\left(1-x_{2} t\right)^{-\alpha_{2}}, \frac{1}{t(1-t)}\right] \\
=\sum_{m_{1}, m_{2}=0}^{\infty} \frac{(\alpha)_{m_{1}+m_{2}}\left(\alpha_{1}\right)_{m_{1}}\left(\alpha_{2}\right)_{m_{2}} x_{1}^{m_{1}} x_{2}^{m_{2}}}{(\alpha+\beta)_{m_{1}+m_{2}} m_{1} ! m_{2} !} \\
\times_{r+2} F_{t+2}\left[\begin{array}{c}
\left(f_{r}\right), \frac{1}{2}\left(1-\alpha-\beta-m_{1}-m_{2}\right), 1-\frac{1}{2}\left(\alpha+\beta+m_{1}+m_{2}\right) ; \\
\left(g_{t}\right), 1-\left(\alpha+m_{1}+m_{2}\right), 1-\beta ;
\end{array}\right] \\
\left(\operatorname{Re}(\alpha), \operatorname{Re}(\beta)>0 ; \max \left\{\left|x_{1}\right|,\left|x_{2}\right|\right\}<1\right) .
\end{gathered}
$$

Case 2. Taking $\phi(t)={ }_{p} F_{q}\left[\begin{array}{l}\left(a_{p}\right) ; \\ \left(b_{q}\right) ;\end{array}{ }^{c t(e-t)}\right], \psi(t)=1 / t(e-t)$, $a=0$, and $\gamma=1$ in (15) and using [7, page 303 (1)]

$$
\begin{gathered}
\left.\int_{0}^{e} t^{\alpha-1}(e-t)^{\beta-1}{ }_{p} F_{q}\left[\begin{array}{l}
\left(a_{p}\right) ; \\
\left(b_{q}\right) ;
\end{array}\right]+(e-t)\right] d t \\
=(e)^{\alpha+\beta-1} B(\alpha, \beta) \\
\times{ }_{p+2} F_{q+2}\left[\begin{array}{c}
\left(a_{p}\right), \alpha, \beta ; \\
\left(b_{q}\right), \frac{\alpha+\beta}{2}, \frac{\alpha+\beta+1}{2} ;
\end{array}\right] \\
\left(e, \operatorname{Re}(\alpha), \operatorname{Re}(\beta)>0 ;\left|\arg \left(4-c e^{2}\right)\right|<\pi\right),
\end{gathered}
$$

we find the following integral:

$$
\begin{aligned}
& I_{d, \alpha, \beta, 1,0, e}\left[{ } _ { p } F _ { q } \left[\begin{array}{ll}
\left(a_{p}\right) ; & \left.c t(e-t)], \frac{1}{t(e-t)}\right] \\
\left(b_{q}\right) ; &
\end{array}\right.\right. \\
&=(e)^{\alpha+\beta-1} \sum_{m=0}^{\infty} \frac{\left(a_{p}\right)_{m}(\alpha)_{m}(\beta)_{m}\left(c e^{2} / 4\right)^{m}}{\left(b_{q}\right)_{m}((\alpha+\beta) / 2)_{m}((\alpha+\beta+1) / 2)_{m} m !} \\
& \times{ }_{r+2} F_{t+2}\left[\begin{array}{cc}
\left(f_{r}\right), \frac{1}{2}(1-\alpha-\beta)-m, 1-\frac{1}{2}(\alpha+\beta)-m ; & \frac{4 d}{e^{2}}
\end{array}\right] \\
& \quad\left(g_{t}\right), 1-\alpha-m, 1-\beta-m ;\left(e, \operatorname{Re}(\alpha), \operatorname{Re}(\beta)>0 ;\left|\arg \left(4-c e^{2}\right)\right|<\pi\right) .
\end{aligned}
$$

Case 3. Taking $\phi(t)={ }_{p} F_{q}\left[\begin{array}{l}\left(a_{p}\right) ; \\ \left(b_{q}\right) ;\end{array} t(e-t)\right], \psi(t)=1 /\left(t^{2}(e-t)^{2}\right)$, $a=0$, and $\gamma=1$ and proceeding as in Case 2 , we find the following integral:

$$
\begin{aligned}
& I_{d, \alpha, \beta, 1,0, e}\left[{ }_{p} F_{q}\left[\begin{array}{l}
\left(a_{p}\right) ; \\
\left(b_{q}\right) ;
\end{array}\right]\right. \\
& =(e)^{\alpha+\beta-1} \sum_{m=0}^{\infty} \frac{\left(a_{p}\right)_{m}(\alpha)_{m}(\beta)_{m}\left(c e^{2} / 4\right)^{m}}{\left(b_{q}\right)_{m}((\alpha+\beta) / 2)_{m}((\alpha+\beta+1) / 2)_{m} m !}
\end{aligned}
$$




$$
\begin{aligned}
& \times{ }_{r+6} F_{t+8}\left[\begin{array}{c}
\left(f_{r}\right), \frac{j-\alpha-\beta}{2}, \frac{1}{2}(1-\alpha-\beta)-m, 1-\frac{1}{2}(\alpha+\beta)-m ; \\
\left(g_{t}\right), \frac{j-\alpha-\beta}{4}, \frac{1-\alpha-m}{2}, \frac{2-\alpha-m}{2}, \frac{1-\beta-m}{2}, \frac{2-\beta-m}{2} ;
\end{array}\right] \\
& (j=1,2,3,4)\left(e, \operatorname{Re}(\alpha), \operatorname{Re}(\beta)>0 ;\left|\arg \left(4-c e^{2}\right)\right|<\pi\right) .
\end{aligned}
$$

Case 4. Taking $\phi(t)=\mathbf{K}(c \sqrt{t(e-t)}), \psi(t)=1 / t(e-t), a=0$, and $\gamma=1$ in (15), where $\mathbf{K}(z)$ is the complete elliptic integral of the first kind [4, page 35 (32)], defined by

$$
\mathbf{K}(z)=\int_{0}^{\pi / 2} \frac{d \theta}{\sqrt{\left(1-z^{2} \sin ^{2} \theta\right)}}
$$

and using [7, page $265(4)]$

$$
\begin{aligned}
& \int_{0}^{e} t^{\alpha-1}(e-t)^{\beta-1} \mathbf{K}(c \sqrt{t(e-t)}) d t \\
& =\frac{\pi}{2}(e)^{\alpha+\beta-1} B(\alpha, \beta)_{4} F_{3}\left[\begin{array}{c}
\frac{1}{2}, \frac{1}{2}, \alpha, \beta ; \\
1, \frac{\alpha+\beta}{2}, \frac{\alpha+\beta+1}{2} ;
\end{array}\right] \\
& \quad\left(\operatorname{Re}(\alpha), \operatorname{Re}(\beta)>0 ;\left|\arg \left(4-c^{2} e^{2}\right)\right|<\pi\right),
\end{aligned}
$$

we find the following integral:

$$
\begin{aligned}
& I_{d, \alpha, \beta, 1,0, e}\left[\mathbf{K}(c \sqrt{t(e-t)}), \frac{1}{t(e-t)}\right] \\
& =\frac{\pi}{2}(e)^{\alpha+\beta-1} \sum_{m=0}^{\infty} \frac{(1 / 2)_{m}(1 / 2)_{m}(\alpha)_{m}(\beta)_{m}\left(c^{2} e^{2} / 4\right)^{m}}{(1)_{m}((\alpha+\beta) / 2)_{m}((\alpha+\beta+1) / 2)_{m} m !} \\
& \quad \times{ }_{r+2} F_{t+2}\left[\begin{array}{cc}
\left(f_{r}\right), \frac{1}{2}(1-\alpha-\beta)-m, 1-\frac{1}{2}(\alpha+\beta)-m ; & \left.\frac{4 d}{e^{2}}\right] \\
\left(g_{t}\right), 1-\alpha-m, 1-\beta-m ; & \\
& \left(\operatorname{Re}(\alpha), \operatorname{Re}(\beta)>0 ;\left|\arg \left(4-c^{2} e^{2}\right)\right|<\pi\right) .
\end{array}\right.
\end{aligned}
$$

Case 5. Taking $\phi(t)=\mathbf{E}(c \sqrt{t(e-t)}), \psi(t)=1 / t(e-t), a=0$, and $\gamma=1$ in (15), where $\mathbf{E}(z)$ is the complete elliptic integral of the second kind [4, page 35 (33)], defined by

$$
\mathbf{E}(z)=\int_{0}^{\pi / 2} \sqrt{\left(1-z^{2} \sin ^{2} \theta\right)} d \theta
$$

and using [7, page 280 (3)]

$$
\begin{aligned}
& \int_{0}^{e} t^{\alpha-1}(e-t)^{\beta-1} \mathbf{E}(c \sqrt{t(e-t)}) d t \\
& =\frac{\pi}{2}(e)^{\alpha+\beta-1} B(\alpha, \beta)_{4} F_{3}\left[\begin{array}{c}
-\frac{1}{2}, \frac{1}{2}, \alpha, \beta ; \\
1, \frac{\alpha+\beta}{2}, \frac{\alpha+\beta+1}{2} ;
\end{array}\right] \\
& \quad\left(e, \operatorname{Re}(\alpha), \operatorname{Re}(\beta)>0 ;\left|\arg \left(4-c^{2} e^{2}\right)\right|<\pi\right),
\end{aligned}
$$

we find the following integral:

$$
\begin{aligned}
& I_{d, \alpha, \beta, 1,0, e}\left[\mathbf{E}(c \sqrt{t(e-t)}), \frac{1}{t(e-t)}\right] \\
& =\frac{\pi}{2}(e)^{\alpha+\beta-1} \sum_{m=0}^{\infty} \frac{(-1 / 2)_{m}(1 / 2)_{m}(\alpha)_{m}(\beta)_{m}\left(c^{2} e^{2} / 4\right)^{m}}{(1)_{m}((\alpha+\beta) / 2)_{m}((\alpha+\beta+1) / 2)_{m} m !} \\
& \quad \times{ }_{r+2} F_{t+2}\left[\begin{array}{cc}
\left(f_{r}\right), \frac{1}{2}(1-\alpha-\beta)-m, 1-\frac{1}{2}(\alpha+\beta)-m ; & \frac{4 d}{e^{2}}
\end{array}\right] \\
& \left(g_{t}\right), 1-\alpha-m, 1-\beta-m ; \\
& \left(e, \operatorname{Re}(\alpha), \operatorname{Re}(\beta)>0 ;\left|\arg \left(4-c^{2} e^{2}\right)\right|<\pi\right) .
\end{aligned}
$$

Case 6. Taking $\phi(t)=\arcsin (c \sqrt{t(e-t)}), \psi(t)=1 / t(e-t)$, $a=0$, and $\gamma=2$ in (15) and using [7, page 173 (151)]

$$
\begin{aligned}
& \int_{0}^{e} t^{\alpha-1}(e-t)^{\beta-1} \arcsin ^{2}(c \sqrt{t(e-t)}) d t \\
& =c^{2}(e)^{\alpha+\beta+1} B(\alpha+1, \beta+1) \\
& \times{ }_{5} F_{4}\left[\begin{array}{cc}
1,1,1, \alpha+1, \beta+1 ; & \frac{c^{2} e^{2}}{4} \\
\frac{3}{2}, 2, \frac{\alpha+\beta+2}{2}, \frac{\alpha+\beta+3}{2} ; &
\end{array}\right] \\
& \left(e>0 ; \operatorname{Re}(\alpha), \operatorname{Re}(\beta)>-1 ;\left|\arg \left(4-c^{2} e^{2}\right)\right|<\pi\right) \text {, }
\end{aligned}
$$


we find the following integral:

$$
\begin{aligned}
& I_{d, \alpha, \beta, 2,0, e}\left[\arcsin (c \sqrt{t(e-t)}), \frac{1}{t(e-t)}\right] \\
& =\frac{\alpha \beta c^{2}(e)^{\alpha+\beta+1}}{(\alpha+\beta)(\alpha+\beta+1)} \\
& \quad \times \sum_{m=0}^{\infty} \frac{(1)_{m}(1)_{m}(1)_{m}(\alpha+1)_{m}(\beta+1)_{m}\left(c^{2} e^{2} / 4\right)^{m}}{(3 / 2)_{m}(2)_{m}((\alpha+\beta+2) / 2)_{m}((\alpha+\beta+3) / 2)_{m} m !} \\
& \quad \times{ }_{r+2} F_{t+2}\left[\begin{array}{cc}
\left(f_{r}\right),-\frac{1}{2}(1+\alpha+\beta)-m,-\frac{1}{2}(\alpha+\beta)-m ; & \left.\frac{4 d}{e^{2}}\right] \\
\left(g_{t}\right),-\alpha-m,-\beta-m ; & \\
\left(e>0 ; \operatorname{Re}(\alpha), \operatorname{Re}(\beta)>-1 ;\left|\arg \left(4-c^{2} e^{2}\right)\right|<\pi\right) .
\end{array}\right.
\end{aligned}
$$

Case 7. Taking $\phi(t)=\exp \left(c^{2} t(e-t)\right) \operatorname{erf}(c \sqrt{t(e-t)}), \psi(t)=$ $1 / t(e-t), a=0$, and $\gamma=1$ in (15) and using [7, page 185 (1)]

$$
\begin{gathered}
\int_{0}^{e} t^{\alpha-1}(e-t)^{\beta-1} \exp \left(c^{2} t(e-t)\right) \operatorname{erf}(c \sqrt{t(e-t)}) d t \\
=\frac{2}{\sqrt{\pi}} c(e)^{\alpha+\beta} B\left(\alpha+\frac{1}{2}, \beta+\frac{1}{2}\right) \\
\times{ }_{3} F_{3}\left[\begin{array}{cc}
1, \alpha+\frac{1}{2}, \beta+\frac{1}{2} ; & \left.\frac{c^{2} e^{2}}{4}\right] \\
\frac{3}{2}, \frac{\alpha+\beta+1}{2}, \frac{\alpha+\beta+2}{2} ; & {\left[e>0 ; \operatorname{Re}(\alpha), \operatorname{Re}(\beta)>-\frac{1}{2}\right),}
\end{array}\right.
\end{gathered}
$$

where $\operatorname{erf}(z)=(2 / \sqrt{\pi}) \operatorname{Erf}(z)$ denotes the error function [4], we find the following integral:

$$
\begin{aligned}
& I_{d, \alpha, \beta, 1,0, e}\left[\exp \left(c^{2} t(e-t)\right) \operatorname{erf}(c \sqrt{t(e-t)}), \frac{1}{t(e-t)}\right] \\
& =\frac{2}{\sqrt{\pi}} c(e)^{\alpha+\beta} \frac{B(\alpha+(1 / 2), \beta+(1 / 2))}{B(\alpha, \beta)} \\
& \quad \times \sum_{m=0}^{\infty} \frac{(1)_{m}(\alpha+(1 / 2))_{m}(\beta+(1 / 2))_{m}\left(c^{2} e^{2} / 4\right)^{m}}{(3 / 2)_{m}((\alpha+\beta+1) / 2)_{m}((\alpha+\beta+2) / 2)_{m} m !} \\
& \times{ }_{r+2} F_{t+2}\left[\begin{array}{c}
\left(f_{r}\right), \frac{1}{2}(1-\alpha-\beta)-m,-\frac{1}{2}(\alpha+\beta)-m ; \\
\left(g_{t}\right), \frac{1}{2}-\alpha-m, \frac{1}{2}-\beta-m ; \\
\left(e>0 ; \operatorname{Re}(\alpha), \operatorname{Re}(\beta)>-\frac{1}{2}\right) .
\end{array}\right.
\end{aligned}
$$

Now, we establish a theorem and apply it to obtain the evaluations of certain integrals in terms of extended beta function.

\section{Integrals in Terms of Extended Beta Function}

Consider an $(r+1)$-variable generating function $G\left(x_{1}, x_{2}, \ldots\right.$, $\left.x_{r} ; t\right)$ which possesses a formal (not necessarily convergent for $t \neq 0$ ) power series expansion in $t$ such that [4, page $80(8)$ ]

$$
G\left(x_{1}, x_{2}, \ldots, x_{r} ; t\right)=\sum_{n=0}^{\infty} c_{n} g_{n}\left(x_{1}, x_{2}, \ldots, x_{r}\right) t^{n}
$$

where each member of the generated set $\left\{g_{n}\left(x_{1}, x_{2}, \ldots\right.\right.$, $\left.\left.x_{r}\right)\right\}_{n=0}^{\infty}$ is independent of $t$ and the coefficient set $\left\{c_{n}\right\}_{n=0}^{\infty}$ may contain the parameters of the set $\left\{g_{n}\left(x_{1}, x_{2}, \ldots, x_{r}\right)\right\}_{n=0}^{\infty}$ but is independent of $x_{1}, x_{2}, \ldots, x_{r}$ and $t$.

Theorem 1. Let the generating function $G\left(x_{1}, x_{2}, \ldots, x_{r} ; t\right)$ defined by (31) be such that $G\left(x_{1}, x_{2}, \ldots, x_{r} ; t(u-a)^{\rho}(c-u)^{\sigma}\right)$ remains uniformly convergent for $u \in(a, c), \rho, \sigma \geq 0$, and $\rho+\sigma>0$. Then

$$
\begin{aligned}
\int_{a}^{c}(u-a)^{\lambda-1}(c-u)^{\mu-\lambda-1} \\
\quad \times G\left(x_{1}, x_{2}, \ldots, x_{r} ; t(u-a)^{\rho}(c-u)^{\sigma}\right) \\
\quad \times \exp \left(-\frac{b(c-a)^{2}}{(u-a)(c-u)}\right) d u \\
=\sum_{n=0}^{\infty}(c-a)^{\mu+(\rho+\sigma) n-1} c_{n} g_{n}\left(x_{1}, x_{2}, \ldots, x_{r}\right) \\
\quad \times B(\lambda+\rho n, \mu-\lambda+\sigma n ; b) t^{n} \\
\quad(\operatorname{Re}(\mu)>\operatorname{Re}(\lambda)>0 ; \operatorname{Re}(b)>0)
\end{aligned}
$$

where $B(x, y ; b)$ is the extended beta function defined by (7).

Proof. Applying the definition of $G\left(x_{1}, x_{2}, \ldots, x_{r} ; t\right)$ given in (31) in the l.h.s. of (32), we get

$$
\begin{aligned}
& \sum_{n=0}^{\infty} c_{n} g_{n}\left(x_{1}, x_{2}, \ldots, x_{r}\right) t^{n} \\
& \quad \times \int_{a}^{c}(u-a)^{\lambda+\rho n-1}(c-u)^{\mu-\lambda+\sigma n-1} \\
& \quad \times \exp \left(-\frac{b(c-a)^{2}}{(u-a)(c-u)}\right) d u,
\end{aligned}
$$

which by using (9) yields the r.h.s. of (32).

Corollary 2. With definition (31) and notations as in Theorem 1, one has

$$
\begin{aligned}
& \int_{0}^{1} u^{\lambda-1}(1-u)^{\mu-\lambda-1} G\left(x_{1}, x_{2}, \ldots, x_{r} ; t u^{\rho}(1-u)^{\sigma}\right) \\
& \quad \times \exp \left(-\frac{b}{u(1-u)}\right) d u
\end{aligned}
$$




$$
\begin{gathered}
=\sum_{n=0}^{\infty} c_{n} g_{n}\left(x_{1}, x_{2}, \ldots, x_{r}\right) B(\lambda+\rho n, \mu-\lambda+\sigma n ; b) t^{n} \\
(\operatorname{Re}(\mu)>\operatorname{Re}(\lambda)>0 ; \operatorname{Re}(b)>0) .
\end{gathered}
$$

Proof. Taking $a=0$ and $c=1$ in (32), we get (34).

Corollary 3. With definition (31) and notations as in Theorem 1, one has

$$
\begin{aligned}
& \int_{-1}^{1}(1+u)^{\lambda-1}(1-u)^{\mu-\lambda-1} \\
& \quad \times G\left(x_{1}, x_{2}, \ldots, x_{r} ; t(1+u)^{\rho}(1-u)^{\sigma}\right) \\
& \quad \times \exp \left(-\frac{4 b}{\left(1-u^{2}\right)}\right) d u \\
& =\sum_{n=0}^{\infty}(2)^{\mu+(\rho+\sigma) n-1} c_{n} g_{n}\left(x_{1}, x_{2}, \ldots, x_{r}\right) \\
& \quad \times B(\lambda+\rho n, \mu-\lambda+\sigma n ; b) t^{n} \\
& (\operatorname{Re}(\mu)>\operatorname{Re}(\lambda)>0 ; \operatorname{Re}(b)>0) .
\end{aligned}
$$

Proof. Taking $a=-1$ and $c=1$ in (32), we get (35).

Corollary 4. With definition (31) and notations as in Theorem 1, one has

$$
\begin{gathered}
\int_{a}^{c}(u-a)^{\lambda-1}(c-u)^{-\lambda-1} G\left(x_{1}, x_{2}, \ldots, x_{r} ; t\left(\frac{c-u}{u-a}\right)^{\sigma}\right) \\
\times \exp \left(-\frac{b(c-a)^{2}}{(u-a)(c-u)}\right) d u \\
=\frac{2}{(c-a) e^{2 b}} \sum_{n=0}^{\infty} c_{n} g_{n}\left(x_{1}, x_{2}, \ldots, x_{r}\right) K_{\lambda-\sigma n}(2 b) t^{n} \\
(\operatorname{Re}(\lambda)>0 ; \operatorname{Re}(b)>0) .
\end{gathered}
$$

Proof. Taking $\mu=0$ and $\rho=-\sigma$ in (32) and making use of relation (10), we get (36).

Now, we apply Theorem 1 to derive the evaluations of certain Euler type integrals in terms of $\operatorname{EBF} B(\alpha, \beta ; b)$. We consider the following cases.

Case 1. Consider the generating function of the multivariable Hermite polynomials $H_{n}^{(1,2, \ldots, r)}\left(x_{1}, x_{2}, \ldots, x_{r}\right)$ [8, page 602 $(20,21)]$

$$
\begin{aligned}
\exp & \left(x_{1} t+x_{2} t^{2}+\cdots+x_{r} t^{r}\right) \\
& =\sum_{n=0}^{\infty} H_{n}^{(1,2, \ldots, r)}\left(x_{1}, x_{2}, \ldots, x_{r}\right) \frac{t^{n}}{n !} .
\end{aligned}
$$

Applying Theorem 1 to generating function (37), we find the following integral:

$$
\begin{aligned}
& \int_{a}^{c}(u-a)^{\lambda-1}(c-u)^{\mu-\lambda-1} \\
& \times \exp \left(-\frac{b(c-a)^{2}}{(u-a)(c-u)}\right. \\
& \left.+\sum_{j=1}^{r} x_{j}\left(t(u-a)^{\rho}(c-u)^{\sigma}\right)^{j}\right) d u \\
& =\sum_{n=0}^{\infty}(c-a)^{\mu+(\rho+\sigma) n-1} H_{n}^{(1,2, \ldots, r)}\left(x_{1}, x_{2}, \ldots, x_{r}\right) \\
& \times B(\lambda+\rho n, \mu-\lambda+\sigma n ; b) \frac{t^{n}}{n !} \\
& (\operatorname{Re}(\mu)>\operatorname{Re}(\lambda)>0 ; \operatorname{Re}(b)>0 ; \rho, \sigma \geq 0 ; \rho+\sigma>0) . \\
& \text { Taking } r=3 \text { in (38), we get } \\
& \int_{a}^{c}(u-a)^{\lambda-1}(c-u)^{\mu-\lambda-1} \\
& \times \exp \left(-\frac{b(c-a)^{2}}{(u-a)(c-u)}\right. \\
& \left.+\sum_{j=1}^{3} x_{j}\left(t(u-a)^{\rho}(c-u)^{\sigma}\right)^{j}\right) d u \\
& =\sum_{n=0}^{\infty}(c-a)^{\mu+(\rho+\sigma) n-1} H_{n}\left(x_{1}, x_{2}, x_{3}\right) \\
& \times B(\lambda+\rho n, \mu-\lambda+\sigma n ; b) \frac{t^{n}}{n !} \\
& (\operatorname{Re}(\mu)>\operatorname{Re}(\lambda)>0 ; \operatorname{Re}(b)>0 ; \rho, \sigma \geq 0 ; \rho+\sigma>0),
\end{aligned}
$$

where $H_{n}\left(x_{1}, x_{2}, x_{3}\right)$ are the 3 -variable Hermite polynomials (3VHP) [9].

Again, taking $r=2$ in (38), we get

$$
\begin{gathered}
\int_{a}^{c}(u-a)^{\lambda-1}(c-u)^{\mu-\lambda-1} \\
\quad \times \exp \left(-\frac{b(c-a)^{2}}{(u-a)(c-u)}\right. \\
\left.\quad+\sum_{j=1}^{2} x_{j}\left(t(u-a)^{\rho}(c-u)^{\sigma}\right)^{j}\right) d u \\
=\sum_{n=0}^{\infty}(c-a)^{\mu+(\rho+\sigma) n-1} H_{n}\left(x_{1}, x_{2}\right) \\
\quad \times B(\lambda+\rho n, \mu-\lambda+\sigma n ; b) \frac{t^{n}}{n !} \\
(\operatorname{Re}(\mu)>\operatorname{Re}(\lambda)>0 ; \operatorname{Re}(b)>0 ; \rho, \sigma \geq 0 ; \rho+\sigma>0),
\end{gathered}
$$


where $H_{n}\left(x_{1}, x_{2}\right)$ are the 2-variable Hermite-Kampé deFériet polynomials (2VHKdFP) [10].

Next, replacing $x_{1}$ by $x, x_{r}$ by $y$ and taking $x_{2}=$ $x_{3}=\cdots=x_{r-1}=0$ in (38) and using the relation $H_{n}^{(1,2, \ldots, r)}(x, 0,0, \ldots, 0, y)=g_{n}^{r}(x, y)$, we get

$$
\begin{gathered}
\int_{a}^{c}(u-a)^{\lambda-1}(c-u)^{\mu-\lambda-1} \\
\times \exp \left(-\frac{b(c-a)^{2}}{(u-a)(c-u)}+x t(u-a)^{\rho}(c-u)^{\sigma}\right. \\
\left.\quad+y\left(t(u-a)^{\rho}(c-u)^{\sigma}\right)^{r}\right) d u \\
=\sum_{n=0}^{\infty}(c-a)^{\mu+(\rho+\sigma) n-1} g_{n}^{r}(x, y) \\
\quad \times B(\lambda+\rho n, \mu-\lambda+\sigma n ; b) \frac{t^{n}}{n !} \\
(\operatorname{Re}(\mu)>\operatorname{Re}(\lambda)>0 ; \operatorname{Re}(b)>0 ; \rho, \sigma \geq 0 ; \rho+\sigma>0),
\end{gathered}
$$

where $g_{n}^{r}(x, y)$ are the Gould-Hopper generalized Hermite polynomials (GHGHP) [11].

Further, replacing $x_{1}$ by $v x$ and taking $x_{2}=x_{3}=\cdots=$ $x_{r-1}=0, x_{r}=-1$ in (38) and using the relation $H_{n}^{(1,2, \ldots, r)}(\nu x$, $0,0, \ldots, 0,-1)=H_{n, r, v}(x)$, we get

$$
\begin{aligned}
& \int_{a}^{c}(u-a)^{\lambda-1}(c-u)^{\mu-\lambda-1} \\
& \quad \times \exp \left(-\frac{b(c-a)^{2}}{(u-a)(c-u)}\right. \\
& \left.\quad+\nu x t(u-a)^{\rho}(c-u)^{\sigma}-\left(t(u-a)^{\rho}(c-u)^{\sigma}\right)^{r}\right) d u \\
& =\sum_{n=0}^{\infty}(c-a)^{\mu+(\rho+\sigma) n-1} H_{n, r, v}(x) \\
& \quad \times B(\lambda+\rho n, \mu-\lambda+\sigma n ; b) \frac{t^{n}}{n !} \\
& (\operatorname{Re}(\mu)>\operatorname{Re}(\lambda)>0 ; \operatorname{Re}(b)>0 ; \rho, \sigma \geq 0 ; \rho+\sigma>0),
\end{aligned}
$$

where $H_{n, r, \nu}(x)$ are the generalized Hermite polynomials [12].

Furthermore, taking $r=v=2$ in (42) and using the relation $H_{n, 2,2}(x)=H_{n}(x)$, we get

$$
\begin{aligned}
\int_{a}^{c}(u-a)^{\lambda-1}(c-u)^{\mu-\lambda-1} \\
\times \exp \left(-\frac{b(c-a)^{2}}{(u-a)(c-u)}\right. \\
\left.\quad+2 x t(u-a)^{\rho}(c-u)^{\sigma}-\left(t(u-a)^{\rho}(c-u)^{\sigma}\right)^{2}\right) d u
\end{aligned}
$$

$$
\begin{gathered}
=\sum_{n=0}^{\infty}(c-a)^{\mu+(\rho+\sigma) n-1} H_{n}(x) \\
\times B(\lambda+\rho n, \mu-\lambda+\sigma n ; b) \frac{t^{n}}{n !} \\
(\operatorname{Re}(\mu)>\operatorname{Re}(\lambda)>0 ; \operatorname{Re}(b)>0 ; \rho, \sigma \geq 0 ; \rho+\sigma>0),
\end{gathered}
$$

where $H_{n}(x)$ are the ordinary Hermite polynomials [1].

Case 2. Consider the generating function of the 2-variable Laguerre polynomials $L_{n}(x, y)$ [13]

$$
\frac{1}{(1-y t)} \exp \left(\frac{-x t}{1-y t}\right)=\sum_{n=0}^{\infty} L_{n}(x, y) t^{n} \quad(|y t|<1),
$$

which can also be expressed as

$$
\exp (y t) C_{0}(x t)=\sum_{n=0}^{\infty} L_{n}(x, y) \frac{t^{n}}{n !}
$$

where $C_{0}(x)$ denotes the 0th-order Tricomi function [4].

Applying Theorem 1 to generating functions (44) and (45), we find the following integrals:

$$
\begin{gathered}
\int_{a}^{c} \frac{(u-a)^{\lambda-1}(c-u)^{\mu-\lambda-1}}{1-y t(u-a)^{\rho}(c-u)^{\sigma}} \\
\times \exp \left(-\frac{b(c-a)^{2}}{(u-a)(c-u)}\right. \\
\left.-\frac{x t(u-a)^{\rho}(c-u)^{\sigma}}{1-y t(u-a)^{\rho}(c-u)^{\sigma}}\right) d u \\
=\sum_{n=0}^{\infty}(c-a)^{\mu+(\rho+\sigma) n-1} L_{n}(x, y) \\
\times B(\lambda+\rho n, \mu-\lambda+\sigma n ; b) t^{n} \\
(\operatorname{Re}(\mu)>\operatorname{Re}(\lambda)>0 ; \operatorname{Re}(b)>0 ; \\
\int_{a}^{c}\left(u t(u-a)^{\rho}(c-u)^{\sigma} \mid<1 ; \rho, \sigma \geq 0 ; \rho+\sigma>0\right), \\
\times \exp \left(-\frac{b(c-a)^{2}}{(u-a)(c-u)}+y t(u-a)^{\rho}(c-u)^{\sigma}\right) \\
\times C_{0}\left(x t(u-a)^{\rho}(c-u)^{\sigma}\right) d u \\
=\sum_{n=0}^{\infty}(c-a)^{\mu+(\rho+\sigma) n-1} L_{n}(x, y) \\
\quad \times B(\lambda+\lambda)>\operatorname{Re}(\lambda)>0 ; \operatorname{Re}(b)>0 ; \rho, \sigma \geq 0 ; \rho+\sigma>0),
\end{gathered}
$$

respectively. 
Taking $y=1$ in (46) and (47) and using the relation $L_{n}(x, 1)=L_{n}(x)$, we get

$$
\begin{aligned}
& \int_{a}^{c} \frac{(u-a)^{\lambda-1}(c-u)^{\mu-\lambda-1}}{1-} t(u-a)^{\rho}(c-u)^{\sigma} \\
& \quad \times \exp \left(-\frac{b(c-a)^{2}}{(u-a)(c-u)}-\frac{x t(u-a)^{\rho}(c-u)^{\sigma}}{1-t(u-a)^{\rho}(c-u)^{\sigma}}\right) d u \\
& =\sum_{n=0}^{\infty}(c-a)^{\mu+(\rho+\sigma) n-1} L_{n}(x) \\
& \quad \times B(\lambda+\rho n, \mu-\lambda+\sigma n ; b) t^{n} \\
& (\operatorname{Re}(\mu)>\operatorname{Re}(\lambda)>0 ; \operatorname{Re}(b)>0 ; \\
& \left.\left|t(u-a)^{\rho}(c-u)^{\sigma}\right|<1 ; \rho, \sigma \geq 0 ; \rho+\sigma>0\right), \\
& \int_{a}^{c}(u-a)^{\lambda-1}(c-u)^{\mu-\lambda-1} \\
& \times \exp \left(-\frac{b(c-a)^{2}}{(u-a)(c-u)}+t(u-a)^{\rho}(c-u)^{\sigma}\right) \\
& \quad \times C_{0}\left(x t(u-a)^{\rho}(c-u)^{\sigma}\right) d u \\
& =\sum_{n=0}^{\infty}(c-a)^{\mu+(\rho+\sigma) n-1} L_{n}(x) \\
& \quad \times B(\lambda+\rho n, \mu-\lambda+\sigma n ; b) \frac{t^{n}}{n !} \\
& \operatorname{Re}(\mu)>\operatorname{Re}(\lambda)>0 ; \operatorname{Re}(b)>0 ; \rho, \sigma \geq 0 ; \rho+\sigma>0),
\end{aligned}
$$

respectively, where $L_{n}(x)$ are the ordinary Laguerre polynomials [1].

Again, taking $y=0$ in (46) and (47) and using the relation $L_{n}(x, 0)=(-x)^{n} / n !$, we get

$$
\begin{aligned}
& \int_{a}^{c}(u-a)^{\lambda-1}(c-u)^{\mu-\lambda-1} \\
& \quad \times \exp \left(-\frac{b(c-a)^{2}}{(u-a)(c-u)}-x t(u-a)^{\rho}(c-u)^{\sigma}\right) d u \\
& =\sum_{n=0}^{\infty}(c-a)^{\mu+(\rho+\sigma) n-1} \\
& \quad \times B(\lambda+\rho n, \mu-\lambda+\sigma n ; b) \frac{(-x t)^{n}}{n !} \\
& (\operatorname{Re}(\mu)>\operatorname{Re}(\lambda)>0 ; \operatorname{Re}(b)>0 ; \rho, \sigma \geq 0 ; \rho+\sigma>0), \\
& \int_{a}^{c}(u-a)^{\lambda-1}(c-u)^{\mu-\lambda-1} \\
& \quad \times \exp \left(-\frac{b(c-a)^{2}}{(u-a)(c-u)}\right) C_{0}\left(x t(u-a)^{\rho}(c-u)^{\sigma}\right) d u \\
& =\sum_{n=0}^{\infty}(c-a)^{\mu+(\rho+\sigma) n-1} \\
& \quad \times B(\lambda+\rho n, \mu-\lambda+\sigma n ; b) \frac{(-x t)^{n}}{(n !)^{2}} \\
& (\operatorname{Re}(\mu)>\operatorname{Re}(\lambda)>0 ; \operatorname{Re}(b)>0 ; \rho, \sigma \geq 0 ; \rho+\sigma>0),
\end{aligned}
$$

respectively.
Next, taking $x=0$ in (46) and (47) and using the relation $L_{n}(0, y)=y^{n}$, we get

$$
\begin{gathered}
\int_{a}^{c} \frac{(u-a)^{\lambda-1}(c-u)^{\mu-\lambda-1}}{1-y t(u-a)^{\rho}(c-u)^{\sigma}} \exp \left(-\frac{b(c-a)^{2}}{(u-a)(c-u)}\right) d u \\
=\sum_{n=0}^{\infty}(c-a)^{\mu+(\rho+\sigma) n-1} \\
\quad \times B(\lambda+\rho n, \mu-\lambda+\sigma n ; b)(y t)^{n} \\
(\operatorname{Re}(\mu)>\operatorname{Re}(\lambda)>0 ; \operatorname{Re}(b)>0 ; \rho, \sigma \geq 0 ; \rho+\sigma>0) \\
\int_{a}^{c}(u-a)^{\lambda-1}(c-u)^{\mu-\lambda-1} \\
\quad \times \exp \left(-\frac{b(c-a)^{2}}{(u-a)(c-u)}+y t(u-a)^{\rho}(c-u)^{\sigma}\right) \\
=\sum_{n=0}^{\infty}(c-a)^{\mu+(\rho+\sigma) n-1} \\
\times B(\lambda+\rho n, \mu-\lambda+\sigma n ; b) \frac{(y t)^{n}}{n !} \\
\operatorname{Re}(\mu)>\operatorname{Re}(\lambda)>0 ; \operatorname{Re}(b)>0 ; \rho, \sigma \geq 0 ; \rho+\sigma>0)
\end{gathered}
$$

respectively. Replacing $y$ by $-x$ in (53), it reduces to (50).

Case 3. Consider the generating function of the HermiteAppell polynomials ${ }_{H} A_{n}\left(x_{1}, x_{2}, x_{3}\right)$ [14, page 759 (2.3)]

$$
A(t) \exp \left(x_{1} t+x_{2} t^{2}+x_{3} t^{3}\right)=\sum_{n=0}^{\infty}{ }_{H} A_{n}\left(x_{1}, x_{2}, x_{3}\right) \frac{t^{n}}{n !}
$$

Applying Theorem 1 to generating function (54), we find the following integral:

$$
\begin{aligned}
\int_{a}^{c}(u-a)^{\lambda-1}(c-u)^{\mu-\lambda-1} A\left(t(u-a)^{\rho}(c-u)^{\sigma}\right) \\
\quad \times \exp \left(-\frac{b(c-a)^{2}}{(u-a)(c-u)}+\sum_{j=1}^{3} x_{j}\left(t(u-a)^{\rho}(c-u)^{\sigma}\right)^{j}\right) d u \\
=\sum_{n=0}^{\infty}(c-a)^{\mu+(\rho+\sigma) n-1} A_{n}\left(x_{1}, x_{2}, x_{3}\right) \\
\quad \times B(\lambda+\rho n, \mu-\lambda+\sigma n ; b) \frac{t^{n}}{n !} \\
\quad(\operatorname{Re}(\mu)>\operatorname{Re}(\lambda)>0 ; \operatorname{Re}(b)>0 ; \rho, \sigma \geq 0 ; \rho+\sigma>0) .
\end{aligned}
$$


Taking $x_{2}=x_{3}=0$ and replacing $x_{1}$ by $x$ in (55) and using the relation ${ }_{H} A_{n}(x, 0,0)=A_{n}(x)[14$, page $760(2.6)]$, we get

$$
\begin{aligned}
& \int_{a}^{c}(u-a)^{\lambda-1}(c-u)^{\mu-\lambda-1} A\left(t(u-a)^{\rho}(c-u)^{\sigma}\right) \\
& \quad \times \exp \left(-\frac{b(c-a)^{2}}{(u-a)(c-u)}+x t(u-a)^{\rho}(c-u)^{\sigma}\right) d u \\
& =\sum_{n=0}^{\infty}(c-a)^{\mu+(\rho+\sigma) n-1} A_{n}(x) \\
& \quad \times B(\lambda+\rho n, \mu-\lambda+\sigma n ; b) \frac{t^{n}}{n !} \\
& (\operatorname{Re}(\mu)>\operatorname{Re}(\lambda)>0 ; \operatorname{Re}(b)>0 ; \rho, \sigma \geq 0 ; \rho+\sigma>0),
\end{aligned}
$$

where $A_{n}(x)$ are the Appell polynomials $[4,15]$.

Case 4. Consider the generating function of the LaguerreAppell polynomials ${ }_{L} A_{n}(x, y)[16]$

$$
\begin{aligned}
A(t) & {\left[\frac{1}{(1-y t)} \exp \left(\frac{-x t}{1-y t}\right)\right] } \\
& =\sum_{n=0}^{\infty}{ }_{L} A_{n}(x, y) t^{n} \quad(|y t|<1),
\end{aligned}
$$

which can also be expressed as follows:

$$
A(t) \exp (y t) C_{0}(x t)=\sum_{n=0}^{\infty}{ }_{L} A_{n}(x, y) \frac{t^{n}}{n !} .
$$

Applying Theorem 1 to generating functions (57) and (58), we find the following integrals:

$$
\begin{aligned}
& \int_{a}^{c}(u-a)^{\lambda-1}(c-u)^{\mu-\lambda-1} \frac{A\left(t(u-a)^{\rho}(c-u)^{\sigma}\right)}{1-y t(u-a)^{\rho}(c-u)^{\sigma}} \\
& \quad \times \exp \left(-\frac{b(c-a)^{2}}{(u-a)(c-u)}-\frac{x t(u-a)^{\rho}(c-u)^{\sigma}}{1-y t(u-a)^{\rho}(c-u)^{\sigma}}\right) d u \\
& =\sum_{n=0}^{\infty}(c-a)^{\mu+(\rho+\sigma) n-1}{ }_{L} A_{n}(x, y) \\
& \quad \times B(\lambda+\rho n, \mu-\lambda+\sigma n ; b) t^{n} \\
& (\operatorname{Re}(\mu)>\operatorname{Re}(\lambda)>0 ; \operatorname{Re}(b)>0 ; \\
& \left.\left|y t(u-a)^{\rho}(c-u)^{\sigma}\right|<1 ; \rho, \sigma \geq 0 ; \rho+\sigma>0\right),
\end{aligned}
$$

$$
\begin{aligned}
\int_{a}^{c}(u-a)^{\lambda-1}(c-u)^{\mu-\lambda-1} A\left(t(u-a)^{\rho}(c-u)^{\sigma}\right) \\
\quad \times \exp \left(-\frac{b(c-a)^{2}}{(u-a)(c-u)}+y t(u-a)^{\rho}(c-u)^{\sigma}\right) \\
\quad \times C_{0}\left(x t(u-a)^{\rho}(c-u)^{\sigma}\right) d u \\
=\sum_{n=0}^{\infty}(c-a)^{\mu+(\rho+\sigma) n-1}{ }_{L} A_{n}(x, y) \\
\quad \times B(\lambda+\rho n, \mu-\lambda+\sigma n ; b) \frac{t^{n}}{n !} \\
(\operatorname{Re}(\mu)>\operatorname{Re}(\lambda)>0 ; \operatorname{Re}(b)>0 ; \rho, \sigma \geq 0 ; \rho+\sigma>0),
\end{aligned}
$$

respectively.

Taking $y=0$ and replacing $x$ by $-x$ in (59) and (60) and using the relation ${ }_{L} A_{n}(x, 0)=A_{n}(-x) / n$ ! [16, page $9(2.8)$ ], we get (56) and

$$
\begin{aligned}
& \int_{a}^{c}(u-a)^{\lambda-1}(c-u)^{\mu-\lambda-1} A\left(t(u-a)^{\rho}(c-u)^{\sigma}\right) \\
& \quad \times \exp \left(-\frac{b(c-a)^{2}}{(u-a)(c-u)}\right) C_{0}\left(-x t(u-a)^{\rho}(c-u)^{\sigma}\right) d u \\
& =\sum_{n=0}^{\infty}(c-a)^{\mu+(\rho+\sigma) n-1} A_{n}(x) \\
& \quad \times B(\lambda+\rho n, \mu-\lambda+\sigma n ; b) \frac{t^{n}}{(n !)^{2}} \\
& (\operatorname{Re}(\mu)>\operatorname{Re}(\lambda)>0 ; \operatorname{Re}(b)>0 ; \rho, \sigma \geq 0 ; \rho+\sigma>0),
\end{aligned}
$$

respectively.

Case 5. Consider the generating function of the HermiteSheffer polynomials ${ }_{H_{n}} s_{1}\left(x_{1}, x_{2}, x_{3}\right)$ [17]

$$
\begin{aligned}
A(t) & \exp \left(x_{1} H(t)+x_{2} H^{2}(t)+x_{3} H^{3}(t)\right) \\
& =\sum_{n=0}^{\infty}{ }_{H} s_{n}\left(x_{1}, x_{2}, x_{3}\right) \frac{t^{n}}{n !} .
\end{aligned}
$$

Applying Theorem 1 to generating function (62), we find the following integral:

$$
\begin{aligned}
\int_{a}^{c}(u-a)^{\lambda-1}(c-u)^{\mu-\lambda-1} A\left(t(u-a)^{\rho}(c-u)^{\sigma}\right) \\
\times \exp \left(-\frac{b(c-a)^{2}}{(u-a)(c-u)}\right. \\
\left.+\sum_{j=1}^{3} x_{j} H^{j}\left(t(u-a)^{\rho}(c-u)^{\sigma}\right)\right) d u
\end{aligned}
$$




$$
\begin{gathered}
=\sum_{n=0}^{\infty}(c-a)^{\mu+(\rho+\sigma) n-1}{ }_{H} s_{n}\left(x_{1}, x_{2}, x_{3}\right) \\
\times B(\lambda+\rho n, \mu-\lambda+\sigma n ; b) \frac{t^{n}}{n !} \\
(\operatorname{Re}(\mu)>\operatorname{Re}(\lambda)>0 ; \operatorname{Re}(b)>0 ; \rho, \sigma \geq 0 ; \rho+\sigma>0) .
\end{gathered}
$$

Taking $x_{2}=x_{3}=0$ and replacing $x_{1}$ by $x$ in (63) and using the relation ${ }_{H} s_{n}(x, 0,0)=s_{n}(x)[17$, page $16(2.3)]$, we get

$$
\begin{aligned}
& \int_{a}^{c}(u-a)^{\lambda-1}(c-u)^{\mu-\lambda-1} A\left(t(u-a)^{\rho}(c-u)^{\sigma}\right) \\
& \quad \times \exp \left(-\frac{b(c-a)^{2}}{(u-a)(c-u)}+x H\left(t(u-a)^{\rho}(c-u)^{\sigma}\right)\right) d u \\
& =\sum_{n=0}^{\infty}(c-a)^{\mu+(\rho+\sigma) n-1} s_{n}(x) \\
& \quad \times B(\lambda+\rho n, \mu-\lambda+\sigma n ; b) \frac{t^{n}}{n !} \\
& \quad(\operatorname{Re}(\mu)>\operatorname{Re}(\lambda)>0 ; \operatorname{Re}(b)>0 ; \rho, \sigma \geq 0 ; \rho+\sigma>0),
\end{aligned}
$$

where $s_{n}(x)$ are the Sheffer polynomials $[15,18,19]$.

\section{Concluding Remarks}

In this paper, we have derived the evaluations of certain Euler type integrals. We have also established a theorem and applied it to obtain evaluations of some integrals in terms of EBF $B(\alpha, \beta ; b)$. We remark that these results can be extended to multivariable case.

To give an example, we consider the following integral representation [5, page 965 (20)] of Lauricella's multiple hypergeometric series $F_{D}^{(n)}[4]$ :

$$
\begin{aligned}
& \int_{0}^{1} t^{\alpha-1}(1-t)^{\beta-1} \prod_{i=1}^{n}\left(1-x_{i} t\right)^{-\alpha_{i}} d t \\
& \quad=B(\alpha, \beta) F_{D}^{(n)}\left[\alpha, \alpha_{1}, \alpha_{2}, \ldots, \alpha_{n} ; \alpha+\beta ; x_{1}, x_{2}, \ldots, x_{n}\right] \\
& \left(\operatorname{Re}(\alpha), \operatorname{Re}(\beta)>0 ; \max \left\{\left|x_{1}\right|,\left|x_{2}\right|, \ldots,\left|x_{n}\right|\right\}<1\right) .
\end{aligned}
$$

Now, taking $\phi(t)=\prod_{i=1}^{n}\left(1-x_{i} t\right)^{-\alpha_{i}}, \psi(t)=1 / t(1-t)$, $a=0$, and $e=\gamma=1$ in (15), using integral (65) in the r.h.s. after expanding ${ }_{r} F_{t}\left[\begin{array}{l}\left.{ }_{\left(f_{r}\right)}\right) ; \\ \left(g_{t}\right) ;\end{array}\right.$ get

$$
\begin{aligned}
& I_{d, \alpha, \beta, 1,0,1}\left[\prod_{i=1}^{n}\left(1-x_{i} t\right)^{-\alpha_{i}}, \frac{1}{t(1-t)}\right] \\
& =\sum_{m_{1}, m_{2}, \ldots, m_{n}=0}^{\infty} \frac{(\alpha)_{m_{1}+m_{2}+\cdots+m_{n}}\left(\alpha_{1}\right)_{m_{1}}\left(\alpha_{2}\right)_{m_{2}} \cdots\left(\alpha_{n}\right)_{m_{n}} \frac{x_{1}^{m_{1}}}{m_{1} !} \frac{x_{2}^{m_{2}}}{m_{2} !} \cdots \frac{x_{n}^{m_{n}}}{m_{n} !}}{(\alpha+\beta)_{m_{1}+m_{2}+\cdots+m_{n}}} \\
& \quad \times f_{r+2} F_{t+2}\left[\begin{array}{c}
\left(f_{r}\right), \frac{1}{2}\left(1-\alpha-\beta-\sum_{i=1}^{n} m_{i}\right), 1-\frac{1}{2}\left(\alpha+\beta+\sum_{i=1}^{n} m_{i}\right) ; \\
\left(g_{t}\right), 1-\left(\alpha+\sum_{i=1}^{n} m_{i}\right), 1-\beta ;
\end{array}\right]
\end{aligned}
$$

$\left(\operatorname{Re}(\alpha), \operatorname{Re}(\beta)>0 ; \max \left\{\left|x_{1}\right|,\left|x_{2}\right|, \ldots,\left|x_{n}\right|\right\}<1\right)$.

We note that, for $\alpha_{3}=\alpha_{4}=\cdots=0$, (66) reduces to (17).

Further, we extend Theorem 1 as follows.

Theorem 5. Let the conditions for $G\left(x_{1}, x_{2}, \ldots, x_{r} ; t\right)$ defined by (31) be the same as in Theorem 1, and then one has

$$
\begin{aligned}
& \int_{a}^{c}(u-a)^{\lambda-1}(c-u)^{\mu-\lambda-1} \\
& \times \prod_{i=1}^{s}\left(1-y_{i}(u-a)\right)^{-\alpha_{i}} \\
& \quad \times G\left(x_{1}, x_{2}, \ldots, x_{r} ; t(u-a)^{\rho}(c-u)^{\sigma}\right)
\end{aligned}
$$

$$
\times \exp \left(-\frac{b(c-a)^{2}}{(u-a)(c-u)}\right) d u
$$$$
=\sum_{n, m_{1}, m_{2}, \ldots, m_{s}=0}^{\infty}(c-a)^{\mu+(\rho+\sigma) n+m_{1}+m_{2}+\cdots+m_{s}-1}
$$$$
\times c_{n} g_{n}\left(x_{1}, x_{2}, \ldots, x_{r}\right)
$$$$
\times B\left(\lambda+\rho n+\sum_{i=1}^{s} m_{i}, \mu-\lambda+\sigma n ; b\right) \prod_{i=1}^{s} \frac{\left(\alpha_{i}\right)_{m_{i}} y_{i}^{m_{i}}}{m_{i} !} t^{n}
$$$$
(\operatorname{Re}(\mu)>\operatorname{Re}(\lambda)>0 ; \operatorname{Re}(b)>0 ; \rho, \sigma \geq 0 ; \rho+\sigma>0) \text {. }
$$ 
The proof of this theorem is similar to that of Theorem 1 . The following are the consequences of Theorem 5 .

Corollary 6. Let the conditions for $G\left(x_{1}, x_{2}, \ldots, x_{r} ; t\right)$ defined by (31) be the same as in Theorem 1, and then for $b=0$ and $s=2$, one has

$$
\begin{aligned}
& \int_{a}^{c}(u-a)^{\lambda-1}(c-u)^{\mu-\lambda-1} \\
& \times \prod_{i=1}^{2}\left(1-y_{i}(u-a)\right)^{-\alpha_{i}} \\
& \quad \times G\left(x_{1}, x_{2}, \ldots, x_{r} ; t(u-a)^{\rho}(c-u)^{\sigma}\right) d u \\
& =\sum_{n=0}^{\infty}(c-a)^{\mu+(\rho+\sigma) n-1} c_{n} g_{n}\left(x_{1}, x_{2}, \ldots, x_{r}\right) \\
& \quad \times B(\lambda+\rho n, \mu-\lambda+\sigma n) \\
& \times F_{1}\left[\lambda+\rho n, \alpha_{1}, \alpha_{2} ; \mu+(\rho+\sigma) n ;\right. \\
& \left.\quad(c-a) y_{1},(c-a) y_{2}\right] t^{n} \\
& (\operatorname{Re}(\mu)>\operatorname{Re}(\lambda)>0 ; \rho, \sigma \geq 0 ; \rho+\sigma>0) .
\end{aligned}
$$

Corollary 7. Let the conditions for $G\left(x_{1}, x_{2}, \ldots, x_{r} ; t\right)$ defined by (31) be the same as in Theorem 1, and then for $b=0$ and $\rho=\sigma=1$, one has

$$
\begin{gathered}
\int_{a}^{c}(u-a)^{\lambda-1}(c-u)^{\mu-\lambda-1} \\
\times \prod_{i=1}^{s}\left(1-y_{i}(u-a)\right)^{-\alpha_{i}} \\
\quad \times G\left(x_{1}, x_{2}, \ldots, x_{r} ; t(u-a)(c-u)\right) d u \\
=B(\lambda, \mu-\lambda) \sum_{n=0}^{\infty}(c-a)^{\mu+2 n-1} c_{n} g_{n}\left(x_{1}, x_{2}, \ldots, x_{r}\right) \\
\times \frac{(\lambda)_{n}(\mu-\lambda)_{n}}{(\mu)_{2 n}} \\
\times F_{D}^{(s)}\left[\lambda+n, \alpha_{1}, \alpha_{2}, \ldots, \alpha_{s} ; \mu+2 n ;(c-a) y_{1},\right. \\
\left.(c-a) y_{2}, \ldots,(c-a) y_{s}\right] t^{n} \\
(\operatorname{Re}(\mu)>\operatorname{Re}(\lambda)>0) .
\end{gathered}
$$

Example 8. Applying Corollary 7 to generating function (37) of the multivariable Hermite polynomials $H_{n}^{(1,2, \ldots, r)}\left(x_{1}, x_{2}\right.$, $\ldots, x_{r}$ ), we get

$$
\begin{aligned}
& \int_{a}^{c}(u-a)^{\lambda-1}(c-u)^{\mu-\lambda-1} \\
& \times \prod_{i=1}^{s}\left(1-y_{i}(u-a)\right)^{-\alpha_{i}} \\
& \quad \times \exp \left(\sum_{j=1}^{r} x_{j}(t(u-a)(c-u))^{j}\right) d u
\end{aligned}
$$

$$
\begin{gathered}
=B(\lambda, \mu-\lambda) \sum_{n=0}^{\infty}(c-a)^{\mu+2 n-1} H_{n}^{(1,2, \ldots, r)}\left(x_{1}, x_{2}, \ldots, x_{r}\right) \\
\times \frac{(\lambda)_{n}(\mu-\lambda)_{n}}{(\mu)_{2 n}} \\
\times F_{D}^{(s)}\left[\lambda+n, \alpha_{1}, \alpha_{2}, \ldots, \alpha_{s} ; \mu+2 n ;(c-a) y_{1},\right. \\
\left.(c-a) y_{2}, \ldots,(c-a) y_{s}\right] \frac{t^{n}}{n !} \\
(\operatorname{Re}(\mu)>\operatorname{Re}(\lambda)>0) .
\end{gathered}
$$

Remark 9. Taking $a=0$ and $c=1$ in (67), we get an extension of integral (34).

Remark 10. Taking $a=-1$ and $c=1$ in (67), we get an extension of integral (35).

Clearly, it appears that, by applying Theorem 5 and Corollaries 6 and 7 to the generating functions defined by (37), (44), (45), (54), (57), (58), and (62) and also to some other generating functions, a number of interesting integrals can be obtained.

Further, it is remarked that the operational methods provide useful tools to estimate specific theorems in various fields of analysis. This approach requires the validation of any of its consequences by a more rigorous procedure. This is indeed the case of the Ramanujan master theorem [20, 21]. An operational method, already employed to formulate a generalization of the Ramanujan master theorem, is applied for the evaluation of certain integrals by Babusci et al. [22]

The technique adopted in [22] provides a very flexible and powerful tool yielding new results encompassing different aspects of the theory of special functions. The possibility of using the method outlined in [22], for the integrals evaluated in this article, is a further research problem.

\section{Conflict of Interests}

The authors declare that there is no conflict of interests regarding the publication of this paper.

\section{Acknowledgment}

The authors would like to express their thanks to the reviewer(s) for helpful suggestions and comments towards the improvement of this paper.

\section{References}

[1] L. C. Andrews, Special Functions for Engineers and Applied Mathematicians, Macmillan, New York, NY, USA, 1985.

[2] M. A. Chaudhry and S. M. Zubair, "Generalized incomplete gamma functions with applications," Journal of Computational and Applied Mathematics, vol. 55, no. 1, pp. 99-124, 1994.

[3] M. A. Chaudhry, A. Qadir, M. Rafique, and S. M. Zubair, "Extension of Euler's beta function," Journal of Computational and Applied Mathematics, vol. 78, no. 1, pp. 19-32, 1997. 
[4] H. M. Srivastava and H. L. Manocha, A Treatise on Generating Functions, John Wiley \& Sons, New York, NY, USA, 1984.

[5] M. E. H. Ismail and J. Pitman, "Algebraic evaluations of some Euler integrals, duplication formulae for Appell's hypergeometric function $F_{1}$, and Brownian variations," Canadian Journal of Mathematics, vol. 52, no. 5, pp. 961-981, 2000.

[6] J. Pitman, "Brownian motion, bridge, excursion, and meander characterized by sampling at independent uniform times," Electronic Journal of Probability, vol. 11, no. 4, pp. 1-33, 1999.

[7] Y. A. Brychkov, Handbook of Special Functions: Derivatives, Integrals, Series and Other Formulas, CRC Press, New York, NY, USA, 2008.

[8] G. Dattoli, S. Lorenzutta, G. Maino, A. Torre, and C. Cesarano, "Generalized Hermite polynomials and super-Gaussian forms," Journal of Mathematical Analysis and Applications, vol. 203, no. 3, pp. 597-609, 1996.

[9] G. Dattoli, "Generalized polynomials, operational identities and their applications," Journal of Computational and Applied Mathematics, vol. 118, no. 1-2, pp. 111-123, 2000.

[10] P. Appell and J. K. de Fériet, Fonctions Hypergéométriques et Hypersphériques: Polynômes d'Hermite, Gauthier-Villars, Paris, Farnce, 1926.

[11] H. W. Gould and A. T. Hopper, "Operational formulas connected with two generalizations of Hermite polynomials," Duke Mathematical Journal, vol. 29, pp. 51-63, 1962.

[12] M. Lahiri, "On a generalisation of Hermite polynomials," Proceedings of the American Mathematical Society, vol. 27, pp. 117-121, 1971.

[13] G. Dattoli and A. Torre, "Operatorial methods and two variable Laguerre polynomials," Atti della Accademia delle Scienze di Torino. Classe di Scienze Fisiche, Matematiche e Naturali, vol. 132, pp. 1-7, 1998.

[14] S. Khan, G. Yasmin, R. Khan, and N. A. M. Hassan, "Hermitebased Appell polynomials: properties and applications," Journal of Mathematical Analysis and Applications, vol. 351, no. 2, pp. 756-764, 2009.

[15] S. Roman, The Umbral Calculus, Academic Press, New York, NY, USA, 1984.

[16] S. Khan, M. W. Al-Saad, and R. Khan, "Laguerre-based Appell polynomials: properties and applications," Mathematical and Computer Modelling, vol. 52, no. 1-2, pp. 247-259, 2010.

[17] S. Khan, M. W. Al-Saad, and G. Yasmin, "Some properties of Hermite-based Sheffer polynomials," Applied Mathematics and Computation, vol. 217, no. 5, pp. 2169-2183, 2010.

[18] E. D. Rainville, Special Functions, Macmillan, New York, NY, USA, 1960.

[19] E. D. Rainville, SpecialFunctions, Chelsea Publishing, New York, NY, USA, 1971.

[20] B. C. Berndt, Ramanujan's Notebooks-Part I, Springer, New York, NY, USA, 1985.

[21] G. H. Hardy, Ramanujan: Twelve Lectures on Subjects Suggested by His Life and Work, Cambridge University Press, Cambridge, UK, 1940.

[22] D. Babusci, G. Dattoli, G. H. E. Duchamp, K. Górska, and K. A. Penson, "Definite integrals and operational methods," Applied Mathematics and Computation, vol. 219, no. 6, pp. 3017-3021, 2012. 


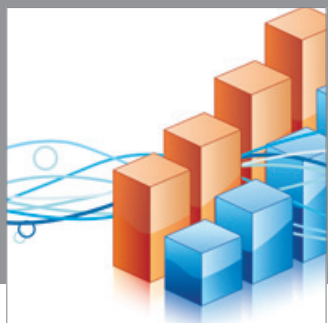

Advances in

Operations Research

mansans

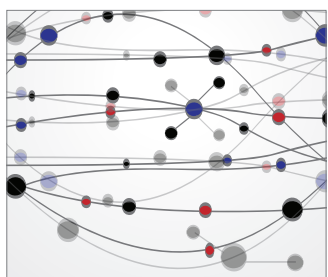

The Scientific World Journal
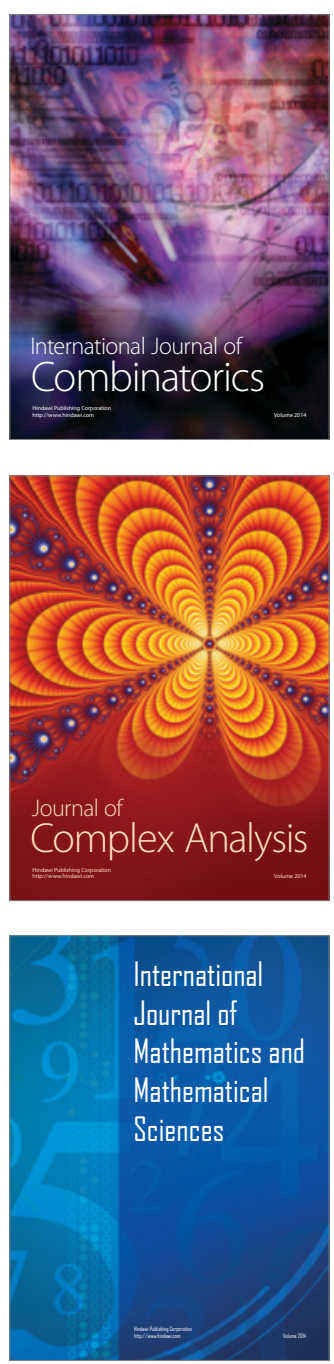
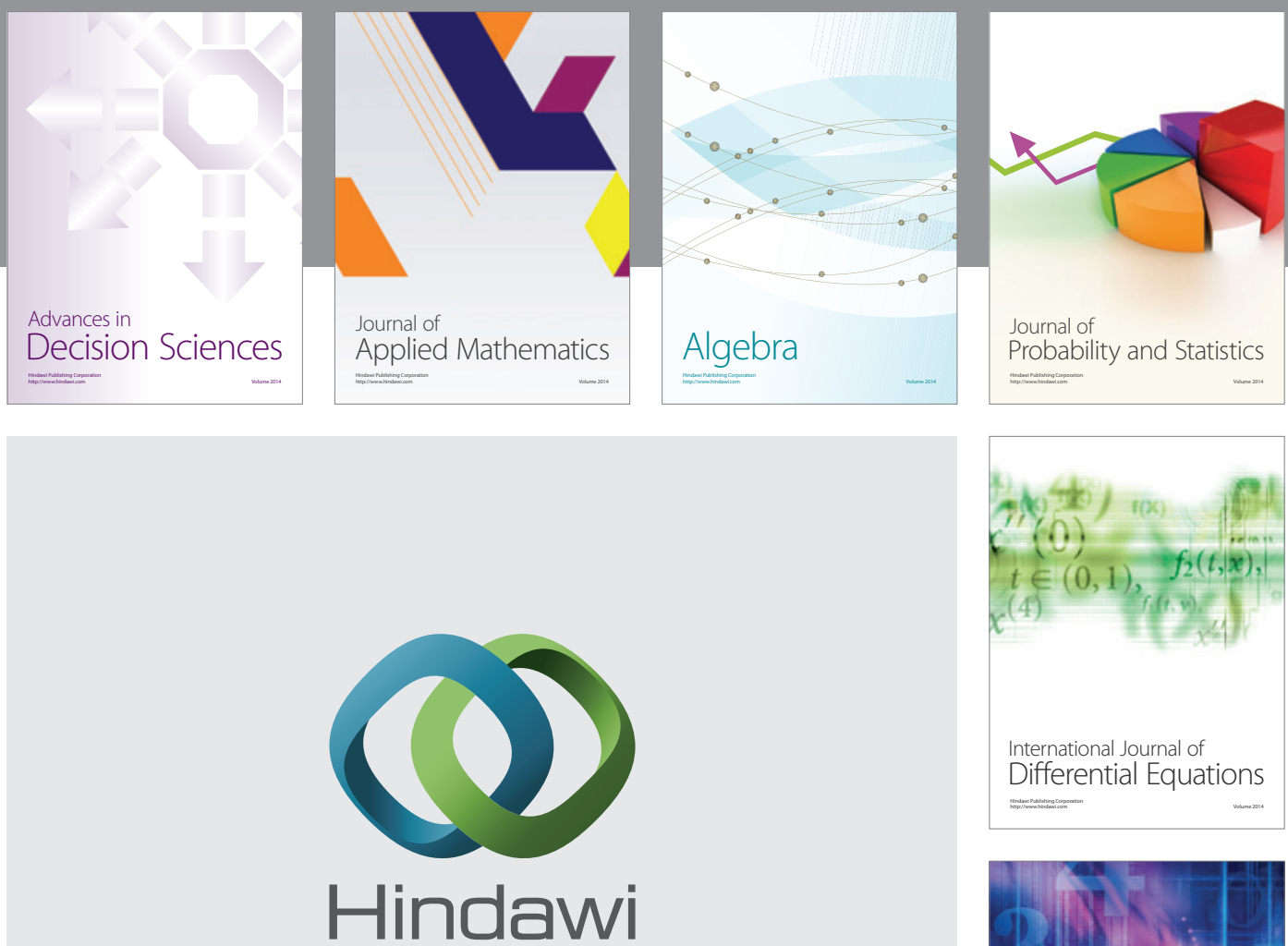

Submit your manuscripts at http://www.hindawi.com
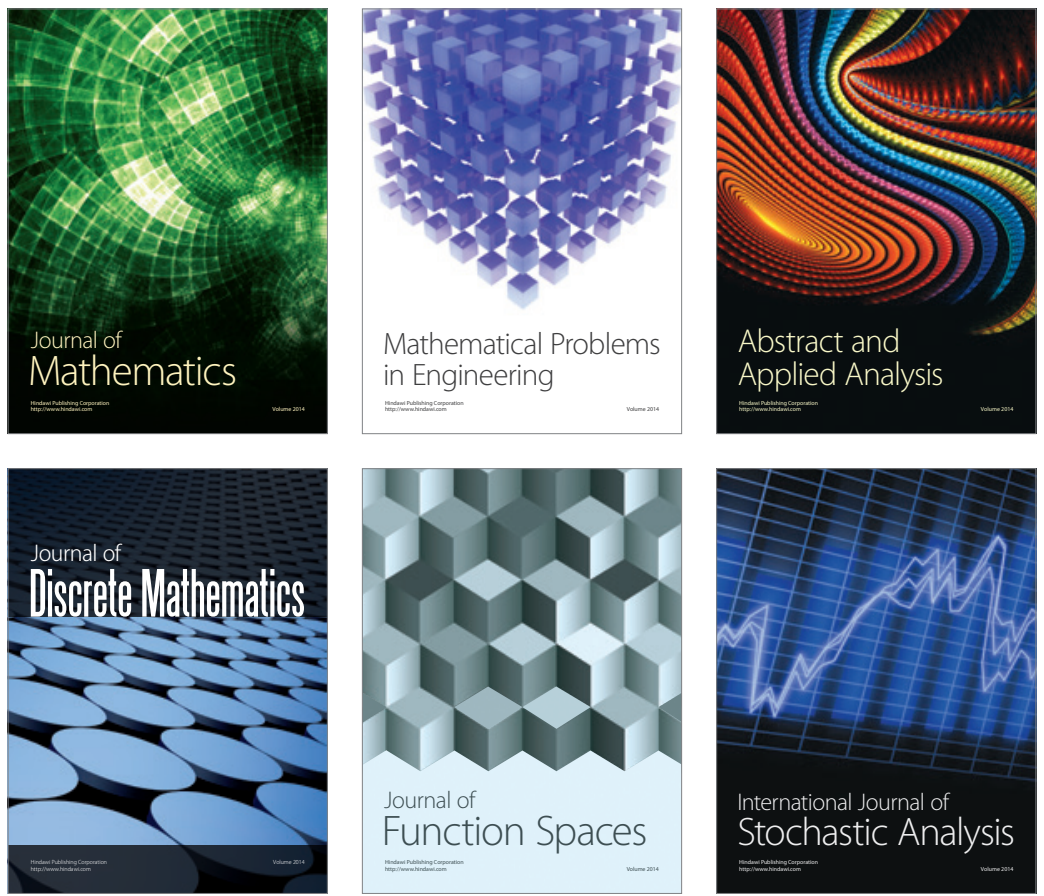

Journal of

Function Spaces

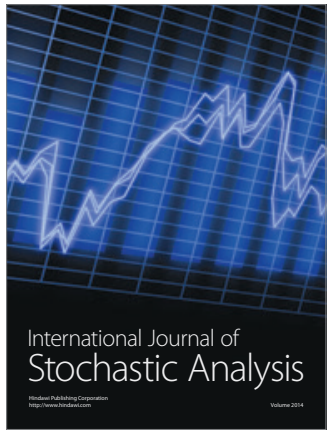

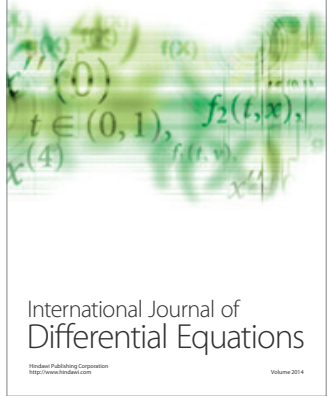
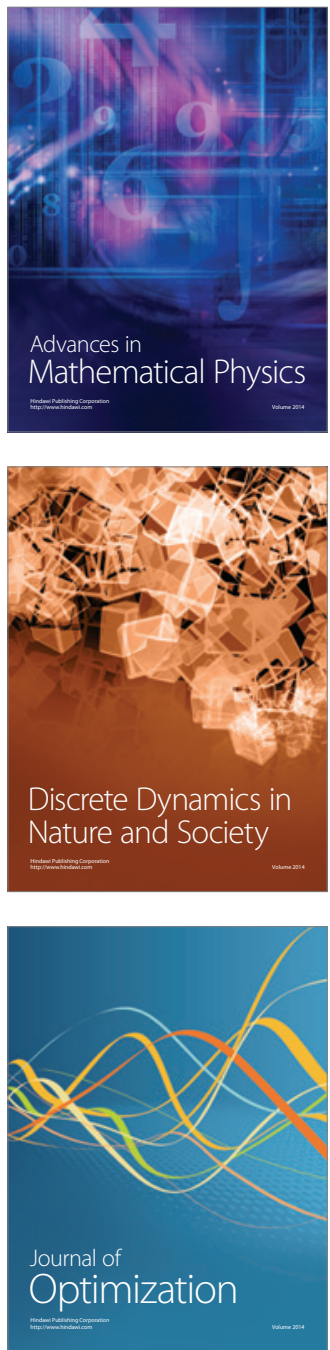